\title{
Application of Transcriptomic Biomarkers in Livestock Product Safety
}

\author{
Xinyong You ${ }^{1,2}$, Qiong $\mathrm{Li}^{1}$ and Yali Zhou ${ }^{1 *}$ \\ ${ }^{1}$ School of Biotechnology and Food Engineering, Anyang Institute of Technology, Anyang, China \\ ${ }^{2}$ Henan Joint International Research Laboratory of Veterinary Biologics Research and Application, Anyang Institute of \\ Technology, Anyang, China
}

*Corresponding author: Yali Zhou, School of Biotechnology and Food Engineering, Anyang Institute of Technology, Anyang, China

ARTICLE INFO

Received: 蔧 August 07, 2019

Published: August 19, 2019

Citation: Xinyong You, Qiong Li, Yali Zhou. Application of Transcriptomic Biomarkers in Livestock Product Safety. Biomed J Sci \& Tech Res 20(4)-2019. BJSTR. MS.ID.003486.

Keywords: Transcriptomics; Biomarker; Livestock Product; Safety

\section{ABSTRACT}

In the research of livestock product quality and safety, the keys are to analyze, screen and confirm illegal additives or contaminants added to livestock products. Searching for biomarkers based on transcriptomics and using them to analyze the physiological changes of animal tissues at the molecular level are becoming a novel and effective approach for monitoring illegal use of contaminants in livestock products. This review included the methods of studying transcriptomic biomarkers and latest progress of applying the biomarkers to livestock product safety detection, aiming at offering new technical measures to screen and detect additives or contaminants illegally added into livestock products so as to guarantee the safety of livestock products.

Abbreviations: PCA: Principal Component Analysis; HCA: Hierarchical Cluster Analysis; PLS-DA: Partial Least Squares Discriminant Analysis; GPs: Growth Promoters; BPSCs: Bovine Prostatic Stromal Cells; TBA: Trenbolone Acetate

\section{Introduction}

The application of illegal additives or contaminants in animal breeding for livestock products severely impacts the development of the livestock industry. Therefore, contaminant analysis and screening become a hot topic among a multitude of researchers at varied levels in different countries. Transcriptomics studies the occurrence and change rules of the transcriptome in biological cells based on the analysis of RNA-level gene expressions. It plays an increasingly important role in studying the physiological activities of tissues or cells and identifying the associations between gene expressions and biological phenomena [1,2]. Biomarkers are quantifiable biological molecules and represent the characteristics of specific physiological states such as in a drug-interfered, normal or pathological process, reflecting the molecular changes caused by interactions between organisms and environmental factors. By identifying and monitoring biomarkers, current biological processes could be diagnosed and monitored in a timely manner. According to previous researches, biomarkers have been successfully applied to molecular medicine, medical diagnosis, disease predication, risk evaluation, food safety, and so on. Identification of biomarkers could be conducted at different molecular levels, such as the genome, epigenome, transcriptome, proteome, metabolome and liposome. Search for biomarkers based on -omics technologies and quantitative determination of contaminants have become one of food safety research focuses in recent years $[3,4]$. Researchers all over the world have carried out biomarker researches targeting at different contaminants in order to monitor the misuse of illegal additives during livestock animal breeding.

\section{Transcriptional Biomarkers}

Transcriptional biomarkers are determined on the transcriptomic platform using large-scale gene expression profiles, qRT-PCR and biostatistical methods such as principal component analysis (PCA), hierarchical cluster analysis (HCA) and partial least squares discriminant analysis (PLS-DA). A standard route for finding and developing gene expression biomarkers is as follows:

a. Setting up a high-quality sample set;

b. Using a large-scale gene expression platform;

c. Analyzing and identifying gene expression biomarkers by mathematics and calculation; 
d. Verifying the capability of the gene expression biomarkers in differentiating independent samples.

Figure 1 shows the workflow of studying transcriptional biomarkers [5]. Identification of gene expression biomarkers in recent years provided a technical platform for studying livestock product safety at different levels (Figure 1).

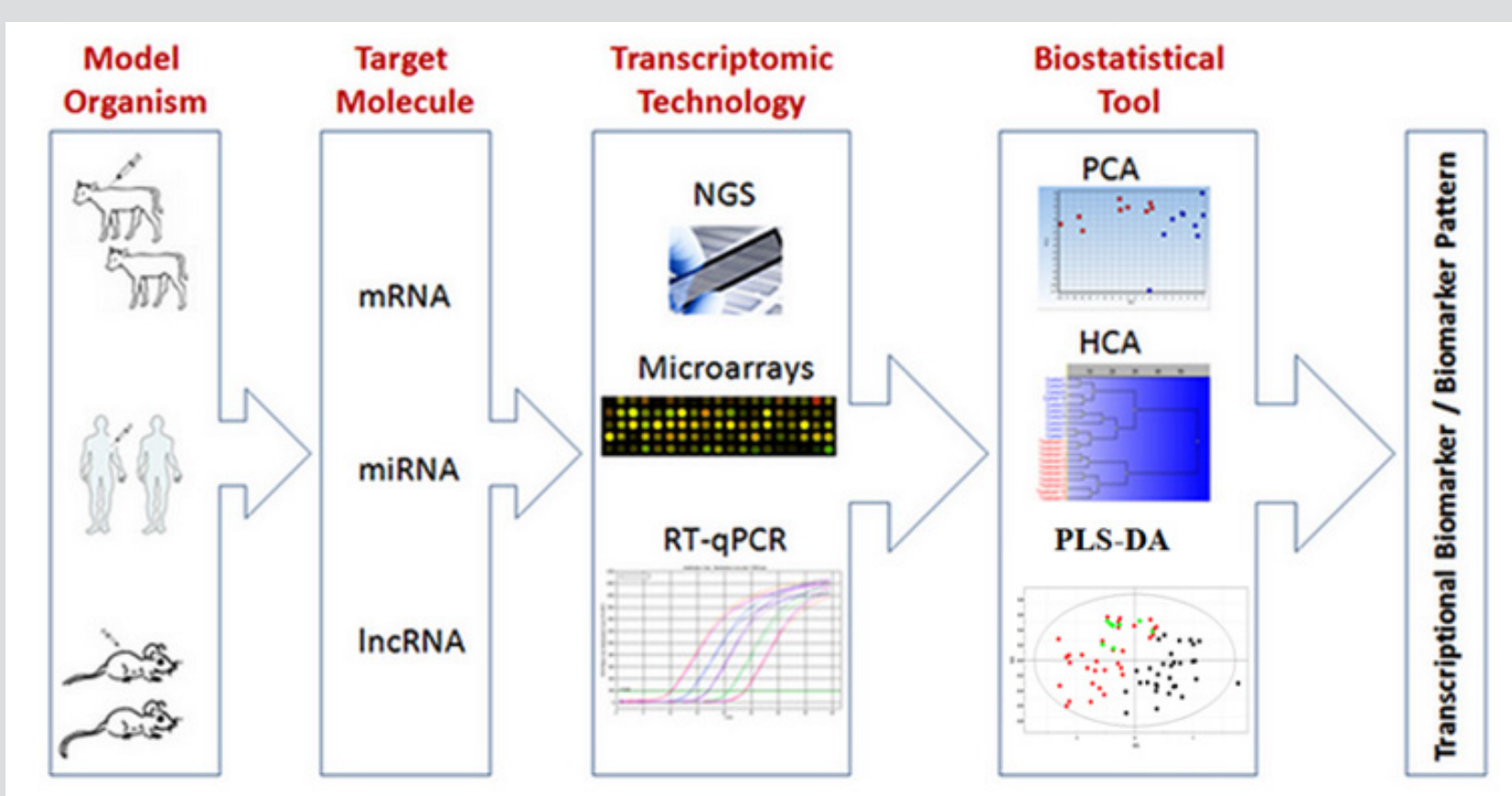

Figure 1: The workflow of transcriptional biomarkers.

Application of Transcriptional Biomarkers in Livestock Product Safety

During livestock breeding, although certain veterinary drugs have been prohibited, new drugs with similar effects and unknown functional mechanisms made drug identification difficult. In search for alternative detection methods, tracking physiological changes of the components in these drugs to find out biomarkers has been a focus. Earlier researches have indicated that the use of illegal growth promoters (GPs) will bring about physiological changes in animals at the molecular level. By analyzing and monitoring gene expression changes in transcriptome using technologies such as gene expression profiles and high-throughput RNA sequencing, target genes in GP-treated animals could be screened, which can serve as a novel and effective screening tool for monitoring illegal use of GPs in animal-sourced food [4,6]. Riedmaier used RNA sequencing to analyze the effects of dimethyl ether acetic acid and estradiol on gene expressions of bovine livers. The expressions of 40 candidate genes were verified by qRT-PCR. Among them, 20 genes were significantly expressed. Then, a biostatistical tool for model identification was employed to analyze the candidate genes, which successfully differentiated samples in the treatment and control groups.

These candidate genes were verified on the wild boars and calves treated by synthesized metabolic agents. Moreover, Riedmaier et al. found common biomarkers in cardiac and liver tissues of cattle. They also determined the candidate genes according to the functional mechanisms of different drugs on these tissues and monitored the mRNA expression levels using fluorescent quantitative PCR. Dynamic PCA was utilized to identify mRNA biomarkers to separate the treatment group from the control group. Therefore, using RNA sequencing, quantitative fluorescent PCR and biostatistical analysis to screen candidate biomarkers have a great potential in detecting illegally synthesized metabolic agents [6,7]. Pegolo studied the transcriptional biomarkers in beef cattle treated with cortical hormone. According to the comprehensive analysis of the DNAmicroarray data of bovine muscle tissues in the control group, the tissues treated by various GPs and those of unknown commercial cattle samples, 73 gene expression biomarkers were found to achieve high-precision classification with the Matthew correlation coefficient of 0.77 , and the percentages of false positives and false negatives were $5 \%$ and $6 \%$, respectively.

Although subject to the animal breed, age and GP treatment, the test results showed that a group of relatively fewer genes could distinguish the control group and cortical hormone treatment group, providing transcriptional biomarkers for effective monitoring of GP misuse in the process of livestock production [8,9]. Maria et al. reported that gene expressions triggered by $17 \beta$-estradiol in bovine prostate could be used to detect abuse of GPs. Quantitative PCR and immunohistochemical methods were employed to analyze the $17 \beta$-estradiol triggered expressions of genes such as associated re- 
ceptors in bovine prostatic stromal cells (BPSCs) and prostate tissues, respectively. It was found that $17 \beta$-estradiol caused significant overexpression of progesterone receptors in both BPSCs and prostate tissues. Gene overexpression could still be detected 15 days after animals were treated by $17 \beta$-estradiol. However, if chemical methods were used, residuals could be detected only several hours after animals were treated, revealing the advantage of PCR assay using $17 \beta$-estradiol [10]. Uslenghi et al. used qPCR to achieve detection of $17 \beta$-estradiol in bull on the basis of progesterone receptors [11]. Divari found that progesterone upregulation could function as a tool for detecting estrogen illegally used in adult beef cattle.

The monitoring of gene regulation at the mRNA level could be used to detect illegal use of synthesized metabolic steroid in meat production. In addition, qPCR was employed to identify illegal use of $17 \beta$-estradiol and dexamethasone based on absolute quantitative determination of oxytocin precursor genes, establishing the sensitivity and specificity of the screening method. This method was a novel screening tool and could significantly increase the success rate of investigating animal food safety $[12,13]$. Starvaggi's research found that the expression of calmodulin in bovine tissues could be used for monitoring illegal use of androgen in beef breeding [14]. Carraro et al. studied the gene expression profiles of the skeletal muscle and liver of bull treated by steroid GPs. By observing the gene expression profile differences and using qRT-PCR, biomarkers could be developed to detect illegal use of dexamethasone in beef cattle production [15]. Cannizzo et al. used morphological check and a transcriptomic approach to identify the use of prednisone in beef cattle and found that a set of clear transcriptional characteristics also supported monitoring of illegal use of drugs in animal husbandry based on biomarkers $[16,17]$.

Elgendy et al. conducted transcriptomic analysis in bovine muscle and liver using both trenbolone acetate (TBA) and $17 \beta$-estradiol (REV), used PCA on the microarray data to identify new candidate biomarkers, and verified the biomarkers using onsite samples, which could well differentiate animals in the control and treated groups [18]. Zhao et al. determined 17 candidate genes by RNA sequencing and literature research. They also used a biostatistical method to obtain six key genes, which could be used as potential transcriptomic biomarkers to monitor abuse of $\beta 2$-receptor drugs in lamb breeding $[19,20]$. You et al. used transcriptomic sequencing and RT-qPCR to screen candidate genes in amantadine-treated chicken breast muscle and liver tissues and preliminarily verified that the candidate genes could be used as biomarkers to monitor illegal use of amantadine drugs in broiler breeding [21].

\section{Conclusion}

Searching for biomarkers based on transcriptomics provides a new approach for identifying illegal additives in livestock products. It has two advantages, which are effective identification of the same type of drugs and gene overexpression detection in the case of a drug residual detection failure by a chemical method. According to the present researches, it is defective using a set of common biomarkers for all types of drugs that are illegally used in animals. A single technique cannot adequately resolve the complexity of the biological system. In contrast, a method integrating various -omics technologies and data could become a key in searching for ideal, stable, long effective and sensitive biomarkers. It is extremely important to use -omics technologies to establish a reliable, specific and sensitive screening method for quick detection of contaminants in livestock products. With gradual progress and development of technologies, analysis of contaminants or illegal additives in livestock products using biomarkers will become increasingly mature.

\section{Acknowledgement}

We are grateful for the support by PhD Research Foundation Project of Anyang Institute of Technology (NO. BSJ2019008), and Henan Joint International Research Laboratory of Veterinary Biologics Research and Application.

\section{Reference}

1. Wang Z, Gerstein M, Snyder M (2009) RNA-Seq: a revolutionary tool for transcriptomics. Nat Rev Genet 10(1): 57-63.

2. Lockhart DJ, Winzeler EA (2000) Genomics, gene expression and DNA arrays. Nature 405(6788): 827-836.

3. Riedmaier I, Becker C, Pfaffl MW, Meyer HHD (2009) The use of omic technologies for biomarker development to trace functions of anabolic agents. J Chromatogr A 1216(46): 8192-8199.

4. Riedmaier I, Pfaffl MW, Meyer HHD (2012) The physiological way: Monitoring RNA expression changes as new approach to combat illegal growth promoter application. Drug Test Anal 4(S1): 70-74.

5. Riedmaier I, Pfaffl MW (2013) Transcriptional biomarkers-high throughput screening, quantitative verification, and bioinformatical validation methods. Methods 59(1): 3-9.

6. Riedmaier I, Benes V, Blake J, Bretschneider N, Zinser C, et al. (2012) RNA-Sequencing as useful screening tool in the combat against the misuse of anabolic agents. Anal Chem 84(15): 6863-6868.

7. Riedmaier I, Spornraft M, Pfaffl MW (2014) Identification of a potential gene expression biomarker signature in bovine liver to detect the abuse of growth promoters. Food Addit Contam A 31(4): 641-649.

8. Pegolo S, Cannizzo FT, Biolatti B, Castagnaro M, Bargelloni L (2014) Transcriptomic profiling as a screening tool to detect trenbolone treatment in beef cattle. Res Vet Sci 96(3): 472-481.

9. Pegolo S, Di CB, Montesissa C, Cannizzo FT, Biolatti B, et al. (2015) Toxicogenomic markers for corticosteroid treatment in beef cattle: Integrated analysis of transcriptomic data. Food Chem Toxicol 77: 1-11.

10. Maria RD, Divari S, Bollo E, Cannizzo FT, Biolatti B, et al. (2009) $17 \beta$-oestradiol-induced gene expression in cattle prostate: biomarkers to detect illegal use of growth promoters. Vet Rec 164(15): 459-464.

11. Uslenghi F, Divari S, Cannizzo FT, Maria RD, Spada F, et al. (2013) Application of absolute qPCR as a screening method to detect illicit $17 \beta$-oestradiol administration in male cattle. Food Addit Contam A 30(2): 253-263.

12. Divari S, Mulasso C, Uslenghi F, Cannizzo FT, Spada F, et al. (2011) Progesterone receptor up-regulation: a diagnostic tool for the illicit use of oestrogens in adult beef cattle. Food Addit Contam A 28(12): 16771686

13. Divari S, Pregel P, Cannizzo FT, Starvaggi CL, Brina N, et al. (2013) Oxytocin precursor gene expression in bovine skeletal muscle is regulated by $17 \beta$-oestradiol and dexamethasone. Food Chem 141(4): 4358-4366. 
14. Starvaggi CL, Biolatti B, Sereno A, Cannizzo FT (2015) Regucalcin expression as a diagnostic tool for the illicit use of steroids in veal calves. J Agr Food Chem 63(23): 5702-5706.

15. Carraro L, Ferraresso S, Cardazzo B, Romualdi C, Montesissa C, et al (2009) Expression profiling of skeletal muscle in young bulls treated with steroidal growth promoters. Physiol Genomics 38(2): 138-148.

16. Cannizzo FT, Pegolo S, Starvaggi CL, Bargelloni L, Divari S, et al (2013) Gene expression profiling of thymus in beef cattle treated with prednisolone. Res Vet Sci 95(2): 540-547.

17. Cannizzo FT, Pegolo S, Pregel P, Manuali E, Salamida S, et al. (2016) Morphological examination and transcriptomic profiling to identify prednisolone treatment in beef cattle. J Agr Food Chem 64(44): 84358446.

\section{ISSN: 2574-1241}

DOI: 10.26717/BJSTR.2019.20.003486

Yali Zhou. Biomed J Sci \& Tech Res

This work is licensed under Creative Commons Attribution 4.0 License

Submission Link: https://biomedres.us/submit-manuscript.php
18. Elgendy R, Giantin M, Montesissa C, Dacasto M (2015) The transcriptome of muscle and liver is responding differently to a combined trenbolone acetate and estradiol implant in cattle. Steroids 106: 1-8.

19. Zhao L, Yang S, Zhang Y, Zhang Y, Hou C, et al. (2016) New analytical tool for the detection of ractopamine abuse in goat skeletal muscle by potential gene expression biomarkers. J Agr Food Chem 64(8): 18611867.

20.Zhao L, Yang S, Cheng Y, Hou C, You X, et al. (2017) Identification of transcriptional biomarkers by RNA-sequencing for improved detection of $\beta 2$-agonists abuse in goat skeletal muscle. Plos One 12(7): e0181695.

21. You X, Xu M, Li Q Zhang K, Hao G, et al. (2019) Discovery of potential transcriptional biomarkers in broiler chicken for detection of amantadine abuse based on RNA sequencing technology. Food Addit Contam A 36(2): 254-269.

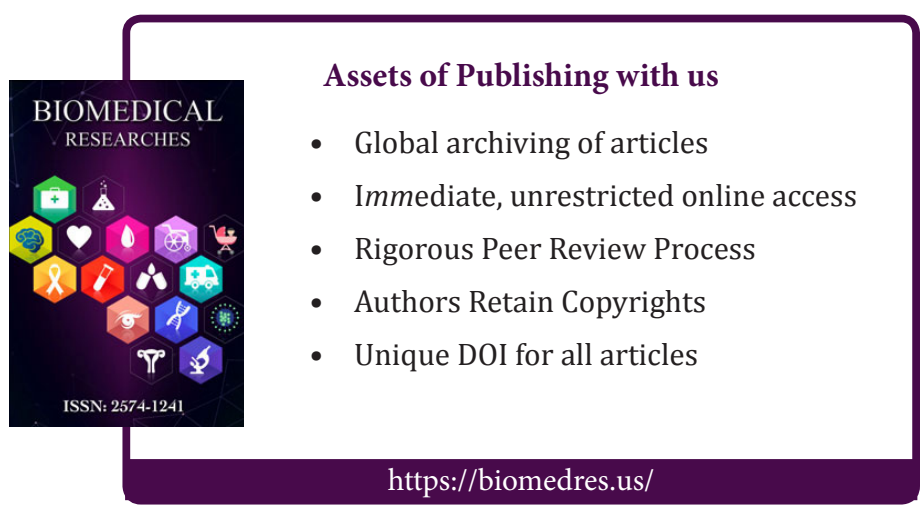

